1999

\title{
Ab initio study of anisotropic magnetism in uranium compounds
}

Eric M. Collins

Nicholas Kioussis

Say Peng Lim

Bernard R. Cooper

Follow this and additional works at: https://researchrepository.wvu.edu/faculty_publications

\section{Digital Commons Citation}

Collins, Eric M.; Kioussis, Nicholas; Lim, Say Peng; and Cooper, Bernard R., "Ab initio study of anisotropic magnetism in uranium compounds" (1999). Faculty Scholarship. 42.

https://researchrepository.wvu.edu/faculty_publications/42 


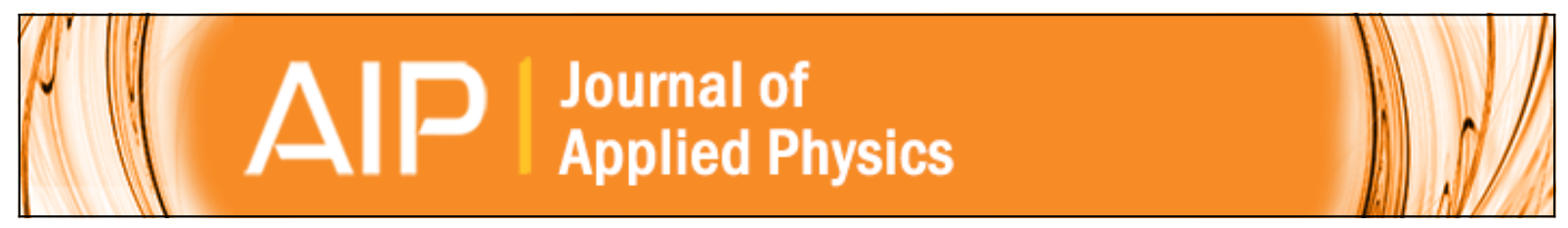

\section{Ab initio study of anisotropic magnetism in uranium compounds}

Eric M. Collins, Nicholas Kioussis, Say Peng Lim, and Bernard R. Cooper

Citation: Journal of Applied Physics 85, 6226 (1999); doi: 10.1063/1.370229

View online: http://dx.doi.org/10.1063/1.370229

View Table of Contents: http://scitation.aip.org/content/aip/journal/jap/85/8?ver=pdfcov

Published by the AIP Publishing

\section{Articles you may be interested in}

Relevance of $4 \mathrm{f}-3 \mathrm{~d}$ exchange to finite-temperature magnetism of rare-earth permanent magnets: An ab-initiobased spin model approach for NdFe12N

J. Appl. Phys. 119, 213901 (2016); 10.1063/1.4952989

Ab initio calculation of anisotropic magnetic properties of complexes. I. Unique definition of pseudospin Hamiltonians and their derivation

J. Chem. Phys. 137, 064112 (2012); 10.1063/1.4739763

Density functional estimations of Heisenberg exchange constants in oligonuclear magnetic compounds:

Assessment of density functional theory versus ab initio

J. Chem. Phys. 131, 224316 (2009); 10.1063/1.3264570

Theoretical study of the magnetic ordering in rare-earth compounds with face-centered-cubic structure J. Appl. Phys. 97, 10A915 (2005); 10.1063/1.1851692

Electronic structure and x-ray magnetic circular dichroism in uranium monochalcogenides Low Temp. Phys. 30, 305 (2004); 10.1063/1.1704618

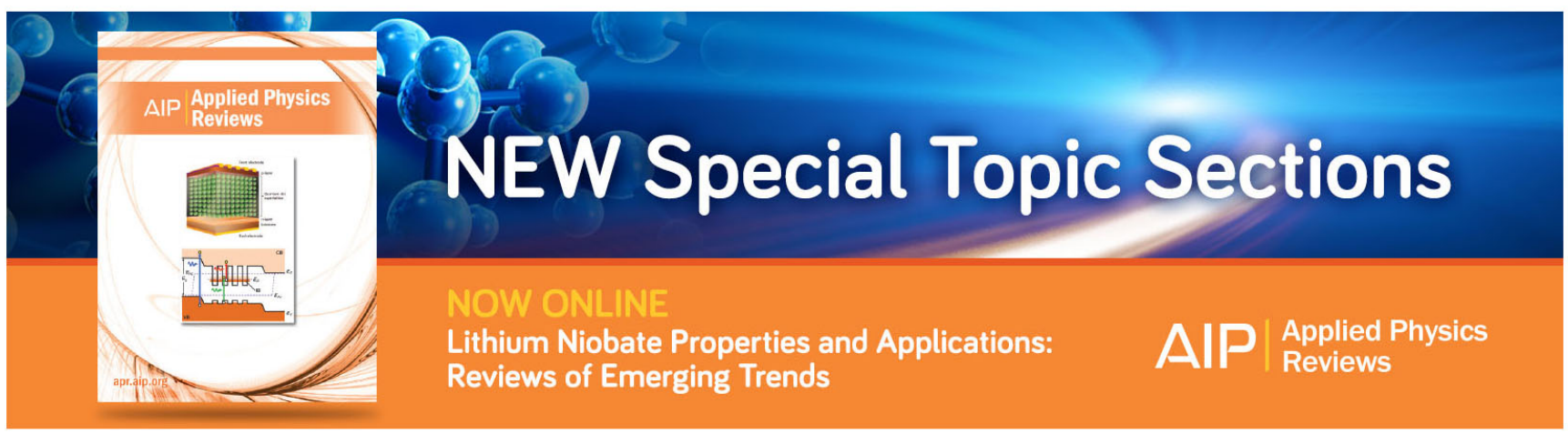




\title{
$A b$ initio study of anisotropic magnetism in uranium compounds
}

\author{
Eric M. Collins, Nicholas Kioussis, ${ }^{\text {a) }}$ and Say Peng Lim \\ Department of Physics and Astronomy, California State University Northridge, Northridge, \\ California 91330-8268
}

\author{
Bernard R. Cooper \\ Department of Physics, West Virginia University, Morgantown, West Virginia 26506
}

\begin{abstract}
We have applied two methods to investigate the origin in the electronic structure of the unusual magnetic behavior of the uranium monochalcogenides and monopnictides. First, we have carried out spin-polarized electronic structure calculations based on the full-potential linearized muffin-tin orbital (FPLMTO) method with only spin-polarization (orbital polarization only via spin-orbit coupling) and also with orbital polarization correction. Second, we have carried out first-principles calculations synthesizing (1) a phenomenological theory of orbitally driven magnetism using a model Hamiltonian which incorporates explicitly the hybridization-induced and Coulomb exchange interactions on an equal footing, and (2) FPLMTO electronic structure calculations allowing a first-principles evaluation of the parameters entering the model Hamiltonian. Within the purely band calculation greater success is obtained for the zero-temperature ordered moments for the more itinerant compounds (US and UP), while the synthesis of phenomenology and electronic structure method gives better agreement with experiment for the more localized pnictides (UBi, USb). Results for the ordered moments and ordering temperatures are presented, and the validity and limitations of the two methods are discussed. (C) 1999 American Institute of Physics.
\end{abstract}

[S0021-8979(99)73308-6]

\section{INTRODUCTION}

The uranium monopnictides $\mathrm{UX}(\mathrm{X}=\mathrm{P}, \mathrm{As}, \mathrm{Sb}, \mathrm{Bi})$ and monochalcogenides $(X=S, S e, T e)$ have become ${ }^{1}$ prototype systems with respect to their unusual magnetic properties and electronic structure. In this class of compounds, with the simple rocksalt structure, one can vary the uranium-uranium spacing $^{2}$ and examine the change in magnetic behavior as the degree of $5 f$-electron localization changes from localized to itinerant behavior, as the chemical environment changes. This family of compounds also allows the study of the dependence of the magnetic behavior on the hybridization between the $5 f$ states of uranium and the uranium $6 d$ states or the ligand $p$ electrons when one replaces the pnictide with its corresponding chalcogenide, where the only apparent chemical change is the addition of a $p$ electron in the anion.

In these systems there is large directional magnetic anisotropy, which changes from the $\langle 001\rangle$ direction in the pnictides to the $\langle 111\rangle$ direction in the chalcogenides. For the monopnictides, both the ordering temperature and ordered moment increase on going down the pnictide column from $\mathrm{UP}\left(125 \mathrm{~K}\right.$ and $\left.1.8 \mu_{B}\right)$ to $\mathrm{UBi}\left(285 \mathrm{~K}\right.$ and $\left.\left.3.0 \mu_{B}\right)\right)^{3}$ On the other hand, for the chalcogenides, while the moment increases on going from US $\left(1.75 \mu_{B}\right)$ to UTe $\left(2.2 \mu_{B}\right)$, the Curie temperature decreases with increasing lattice constant (178 K for US to $104 \mathrm{~K}$ for UTe). ${ }^{4,5}$

To understand the underlying mechanisms responsible for the unusual magnetism in this class of systems, we have employed two methods to calculate the zero-temperature magnetic properties. First, full-potential linear muffin-tin orbital (FPLMTO) electronic structure calculations within the local density approximation (LDA) have been carried out

a)Electronic mail: nkioussi@newton.csun.edu using (i) only spin polarization, with orbital polarization included only through spin-orbit coupling; and (ii) using both spin and orbital polarization correction. ${ }^{6}$ Second, we apply a phenomenological (PHEN) theory of magnetic ordering based on the Anderson and Kondo Hamiltonian, which includes the band- $f$ Coulomb exchange and the band- $f$ hybridization on an equal footing. The parameters entering the phenomenological Hamiltonian are calculated from the FPLMTO electronic structure calculations. These ab initio parameters are then used in a mean field calculation to evaluate the zero-temperature moment and the ordering temperature.

\section{METHOD}

The self-consistent density-functional calculations are based on the FPLMTO method. ${ }^{7}$ Explicit orbital polarization can be taken into account self-consistently by means of an eigenvalue shift, $\Delta V_{m}=-E^{3} L_{z} m_{l}$, at each atom site. ${ }^{6}$ Here, $L_{z}$ is the $z$ component of the total orbital moment of the atom, $m_{l}$ is the magnetic quantum number, and $E^{3}$ is the Racah parameter which is self-consistently evaluated at each iteration.

The phenomenological method is based on the Anderson and Kondo lattice Hamiltonian ${ }^{8}$

$$
H=H_{0}+H_{1},
$$

where

$$
\begin{aligned}
H_{0}= & \sum_{k} \epsilon_{k} b_{k}^{+} b_{k}+\sum_{R m} \epsilon_{m} c_{m}^{+}(R) c_{m}(R) \\
& +\frac{U}{2} \sum_{R, m \neq m^{\prime}} n_{m}(R) n_{m^{\prime}}(R)
\end{aligned}
$$


TABLE I. Values of the calculated and experimental (see Refs. 3-5) magnetic moments for the uranium chalcogenides and pnictides in units of $\mu_{B}$. Listed are the FPLMTO values for the spin moment $\mu_{S}$, the orbital moment $\mu_{L}$, and total moment $\mu$, for the spin polarized only calculation (FP+SP) and for the calculation with spin polarization and orbital polarization correction $(\mathrm{FP}+\mathrm{SP}+\mathrm{OP})$. Also listed are the values of the total magnetic moment of the phenomenological (PHEN) calculations.

\begin{tabular}{|c|c|c|c|c|c|c|c|c|}
\hline & \multicolumn{3}{|c|}{$\mathrm{FP}+\mathrm{SP}$} & \multicolumn{3}{|c|}{$\mathrm{FP}+\mathrm{SP}+\mathrm{OP}$} & \multirow{2}{*}{$\begin{array}{c}\text { PHEN } \\
\mu\end{array}$} & \multirow{2}{*}{$\begin{array}{c}\text { EXPT } \\
\mu\end{array}$} \\
\hline & $\mu_{S}$ & $\mu_{L}$ & $\mu$ & $\mu_{S}$ & $\mu_{L}$ & $\mu$ & & \\
\hline US & -1.87 & 2.39 & 0.52 & -2.06 & 3.58 & 1.51 & 3.1 & 1.6 \\
\hline USe & -2.01 & 2.68 & 0.67 & -2.27 & 3.89 & 1.62 & 3.2 & 2.0 \\
\hline UTe & -2.35 & 3.23 & 0.88 & -2.60 & 4.26 & 1.66 & 3.3 & 2.2 \\
\hline UP & -2.18 & 2.66 & 0.48 & -2.43 & 3.85 & 1.43 & 3.3 & 1.8 \\
\hline UAs & -2.32 & 3.00 & 0.68 & -2.44 & 4.12 & 1.68 & 3.3 & 2.2 \\
\hline USb & -2.37 & 3.50 & 1.13 & -2.60 & 4.57 & 1.97 & 3.3 & 2.8 \\
\hline UBi & -2.55 & 3.82 & 1.28 & -2.69 & 4.79 & 2.10 & 3.2 & 3.0 \\
\hline
\end{tabular}

and

$$
\begin{aligned}
H_{1}= & \sum_{k m R}\left[V_{k m} e^{-i \mathbf{k} \cdot \mathbf{R}} b_{k}^{+} c_{m}(R)+H . C .\right] \\
& +\sum_{k k^{\prime}} \sum_{m m^{\prime} R} J_{m m^{\prime}}\left(\mathbf{k}, \mathbf{k}^{\prime}\right) e^{-i\left(\mathbf{k}-\mathbf{k}^{\prime}\right) \cdot \mathbf{R}} \\
& \times b_{k}^{+} c_{m}^{+}(R) b_{k^{\prime}} c_{m^{\prime}}(R) .
\end{aligned}
$$

The FPLMTO electronic structure calculations are used to evaluate the band energies $\epsilon_{k}$, the $f$-state energy $\epsilon_{m}$, and the on-site Coulomb repulsion U. ${ }^{9}$ Since the $5 f$ states in uranium compounds are more delocalized than the $4 f$ states in cerium, we adopt the "band point of view" scheme to evaluate the hybridization matrix elements $V_{k m} \cdot{ }^{10}$ The band- $f$ exchange Coulomb interaction, $J_{m, m^{\prime}}\left(\mathbf{k}, \mathbf{k}^{\prime}\right)$, is calculated using the more atomiclike scheme. ${ }^{8}$

Having calculated from first principles all parameters in the Hamiltonian, one can then evaluate the two-ion interactions $E_{m_{a} m_{a}^{\prime}}^{m_{b} m_{b}^{\prime}}\left(\mathbf{R}_{\mathbf{b}}-\mathbf{R}_{\mathbf{a}}\right)$ treating $H_{1}$ to second order in perturbation theory. ${ }^{8}$ The exchange interactions contain three types of terms: the pure Coulomb exchange interaction term proportional to $J_{m m^{\prime}}^{2}\left(\mathbf{k}, \mathbf{k}^{\prime}\right)$, the hybridization-mediated exchange interaction term proportional to $V_{k m}^{4}$, and the cross term proportional to $V_{k m}^{2} J_{m m^{\prime}}\left(\mathbf{k}, \mathbf{k}^{\prime}\right)$. These exchange interactions, $E_{m_{a} m_{a}^{\prime}}^{m_{b} m_{b}^{\prime}}\left(\mathbf{R}_{\mathbf{b}}-\mathbf{R}_{\mathbf{a}}\right)$, couple two $f$ ions via singleelectron scattering. The two-ion interactions between the $f^{3}$ multiplets can be determined in terms of the one-electron interactions. ${ }^{10}$ Having obtained the two-ion interactions, the ordering temperature and zero temperature ordered moment can be determined by means of a mean field calculation. ${ }^{9}$

\section{RESULTS AND DISCUSSION}

In Table I we present the calculated values of the zerotemperature uranium magnetic moment in the ferromagnetic state. For the LDA calculations we list the values with and without orbital polarization correction taken into account. To
TABLE II. Characteristic matrix elements of the $10 \times 10$ two-ion interaction matrix $\left(E_{m_{a} m_{a}^{\prime}}^{m_{b} m_{b}^{\prime}}\left(\mathbf{R}_{\mathbf{b}}-\mathbf{R}_{\mathbf{a}}\right)\right)$ of the $f^{3}$ multiplet. Values of the hybridization induced $\left(E_{V^{4}}\right)$, cross terms $\left(E_{V^{2} J}\right)$, and pure Coulomb exchange $\left(E_{J^{2}}\right)$ interactions are listed for the $m=m^{\prime}=5 / 2$ elements for each of the first, second, and third nearest uranium neighbors in units of $K$.

\begin{tabular}{lccc}
\hline \hline & $E_{V^{4}}$ & $E_{V^{2} J}$ & $E_{J^{2}}$ \\
\hline$R=\left(\begin{array}{llll}1 / 2 & 1 / 2 & 0\end{array}\right)$ & 253 & 14.4 & 2.0 \\
$R=\left(\begin{array}{lll}1 & 0 & 0\end{array}\right)$ & 490 & 12.9 & 1.0 \\
$R=\left(\begin{array}{lll}1 & 1 / 2 & 1 / 2\end{array}\right)$ & 29 & 4.2 & 0.1 \\
\hline \hline
\end{tabular}

determine the direction of magnetic anisotropy we have carried out total energy electronic structure calculations with the moment aligned in different directions.

For both the pnictides and chalcogenides we find that the $a b$ initio purely band structure calculations for ferromagnetic configurations give the lowest energy for moment (both spin and orbital) along the $\langle 111\rangle$ direction. The results for the chalcogenides are consistent with experiment, where the easy axis of magnetic anisotropy is along $\langle 111\rangle$; however experimentally, the pnictides are antiferromagnetic with an easy direction of $\langle 001\rangle$. The values of the moments with no orbital polarization correction are consistently lower than the experimental results by a factor of 2 for the heavier pnictides and chalcogenides, and by a factor of 3 for the lighter ones. With orbital polarization correction taken into account the values of the total moment increase and are in better agreement with experiment. These results indicate the importance of the effect of orbital polarization in the uranium compounds. Note, that in all cases the orbital moment is antiparallel (in accordance to Hund's third rule) to and larger than the spin moment. The LDA calculations of the magnetic moments with orbital polarization are in better agreement with experiment for the lighter more itinerant chalcogenides (US) and pnictides (UP). On the other hand, the LDA calculations including the orbital polarization correction fail to give the nearly saturated moments for the heavier uranium compounds which exhibit a more localized nature of the $5 f$ states. Thus, for the more itinerant (lighter) systems where band theory is more appropriate, the refinement of including the orbital polarization corrections is relatively successful in providing agreement with experiment. In contrast, the moments obtained from the phenomenological calculations agree well with experiment for the more-localized $f$-electron pnictides (UBi, USb).

We have calculated the $10 \times 10$ exchange interaction matrix pertinent to the change of magnetic behavior in this class of compounds. Characteristic values of the exchange interaction matrix elements $\left(m=m^{\prime}=5 / 2\right)$ of the $f^{3}$ multiplets for the first three nearest-neighbor shells of US are listed in Table II. The exchange interactions resulting from the hybridization of $f$ states with non- $f$ states are dominant due to the more extended spatial extent of the $5 f$ wave functions. Note, that the second nearest-neighbor exchange interaction is twice as large as the first nearest-neighbor exchange interaction.

Listed in Table III are the calculated ordering temperatures for the phenomenological (PHEN) method, $T_{c}$, as well 
TABLE III. Calculated (from the phenomenological PHEN method) and experimental values of the ordering temperatures for uranium compounds. For the case of the pnictides, the ordering temperature listed is the Néel temperature.

\begin{tabular}{ccc}
\hline \hline & $T_{C}(\mathrm{~K})$ calc & $T_{C}(\mathrm{~K}) \exp$ \\
\hline US & 950 & 178 \\
USe & 580 & 160 \\
UTe & 170 & 104 \\
& & \\
UP & 900 & 125 \\
UAs & 450 & 126 \\
USb & 195 & 241 \\
UBi & 120 & 285 \\
\hline \hline
\end{tabular}

as the experimental values. The synthesis of phenomenology and electronic structure method gives the trend of Curie temperatures for the chalcogenides in qualitative agreement with experiment. We find that the calculated ordering temperatures for the more localized compounds (UBi, USb, UTe) are in better agreement with experiment, while overestimating $T_{c}$ for the more itinerant compounds.

In conclusion, we have applied two different computational methods to study the trend of the low-temperature ordered magnetic moment across the uranium compounds. The method involving pure electronic structure calculations better predicts the moments for the more itinerant systems. On the other hand, the synthesis of phenomenology and electronic structure method provides a better description of the ordered moment for the more localized uranium compounds, and can be used to gain insight into some of the specific contributions of the interactions responsible for the unusual magnetic properties of these compounds. With a quite limited degree of success, the PHEN calculations also give values for the magnetic ordering temperatures. Employing an $a b$ initio based phenomenological approach utilizing the Hub- bard model and a disordered Ising lattice with randomly located holes (two types of uranium sites, embodying the localized and delocalized components, respectively) Cooper and $\operatorname{Lin}^{11}$ obtain the correct values of ordering temperatures and variation with pressure and dilution alloying for the uranium chalcogenides. Recent results for the more-localized cerium pnictides and chalcogenides, based on the synthesis of $a b$ initio and electronic structure calculations, for the ordered magnetic moment and the ordering temperatures are in good agreement with experiment. ${ }^{12}$

\section{ACKNOWLEDGMENTS}

The research at California State University Northridge (CSUN) was supported by the National Science Foundation under Grant No. DMR-9531005, by U.S. Army Grant No. DAAH04-95, and the CSUN Office of Research and Sponsored Projects, and at West Virginia University by the NSF under Grant No. DMR-9120333.

${ }^{1}$ H. Rudiger, H. R. Ott, and O. Vogt, Physica B 130, 538 (1985).

${ }^{2}$ B. Reihl, N. Martensson, and O. Vogt, J. Appl. Phys. 53, 2008 (1982).

${ }^{3}$ S. K. Sinha, G. H. Lander, S. M. Shapiro, and O. Vogt, Phys. Rev. B 23, 4556 (1981)

${ }^{4}$ T. M. Holden, W. J. L. Buyers, E. C. Svensson, J. A. Jackman, A. F. Murray, O. Vogt, and P. de. V. DuPlessis, J. Appl. Phys. 53, 1967 (1982).

${ }^{5}$ T. M. Holden, W. J. L. Buyers, P. de. V. DuPlessis, K. M. Hughes, and M. F. Collins, J. Magn. Magn. Mater. 54-57, 1175 (1986).

${ }^{6}$ O. Eriksson, M. S. S. Brooks, and B. Johansson, Phys. Rev. B 41, 7311 (1990).

${ }^{7}$ D. L. Price and B. R. Cooper, Phys. Rev. B 39, 4945 (1989).

${ }^{8}$ Q. G. Sheng and B. R. Cooper, J. Appl. Phys. 69, 5472 (1991); Phys. Rev. B 50, 965 (1994).

${ }^{9}$ N. Kioussis, B. R. Cooper, and J. M. Wills, Phys. Rev. B 44, 10003 (1991).

${ }^{10}$ Q. G. Sheng, B. R. Cooper, and S. P. Lim, Phys. Rev. B 50, 9215 (1994).

${ }^{11}$ B. R. Cooper and Y. L. Lin, J. Appl. Phys. 83, 6432 (1998) and article EE-06; B. R. Cooper, Y. L. Lin, and Q. G. Sheng, J. Appl. Phys. (these proceedings)

${ }^{12}$ E. M. Collins, N. Kioussis, S. P. Lim, and B. R. Cooper (unpublished). 